\title{
THE COURT OF ELECTORS
}

Ex officio: The President, the Dean, the Registrar

Elected

Professor T. Arie, Drs R. Bluglass, C. P. B. Brook,

Valerie Cowie, K. L. Granville-Grossman, J. A.
Harrington, W. A. Heaton-Ward, M. Markowe, M. Pines, F. Post, Professors K. Rawnsley, W. Linford Rees, Dr A. B. Sclare, Professor W. H. Trethowan, Dr H. H. Wolff:

\section{THE STANDING COMMITTEES}

(The Officers are ex-officio members of all Standing Committees except the

Executive and Finance Committee)

Executive and Finance Committee

Ex-officio: The President, the Registrar, the Treasurer and the Dean

Fellows: Drs W. D. Boyd, J. L. Crammer, J. Harrington, P. G. McGrath, Professor K. Rawnsley, Dr P. Sainsbury.

Members: Drs J. R. Hamilton and Diana Dickens.

\section{Education Committee}

Council Members: Professor T. Arie, Drs M. D. Cashman, Diana Dickens, P. d'Orban, D. Dunne, E. B. Gordon, O. Hill, A. M. P. Kellam, E. G. Lucas, W. R. McKibben, T. Nelson, J. Pedder, R. A. Robinson, K. Schapira, A. Shapiro, A. Smith, W. Lumsden Walker.

Non-Council Members: Wing-Cdr E. Anthony, Drs J. L. T. Birley, L. A. Hersov, Professors J. Hinton, G. F. M. Russell, Surg-Cdr A. W. Scott-Brown, Professor H. J. Walton.

Co-opted Members: Drs Pamela Ashurst (WPWIP), J. A. Betts (AVSC), S. Bhate (OTSC), J. Bowlby (LRLSC), I. G. Bronks (CTSC), J. Cobb (Manpower), D. Gill (OTSC), Mrs B. Nicolas (NSC), Drs Sally Pidd (WPT), T. L. Pilkington (AVSC), Ashley Robin (Manpower), C. P. Seager (NSC), L. Tarlo (CTSC), C. Trotter (WPT).

Observers: Drs E. F. Carr (DHSS), C. Freeman (APIT).

\section{Library Committee}

Council Members: Drs Valerie Cowie, T. L. Dunn, A. B. Sclare, A. Shapiro, E. W. Shepherd, A. C. Smith.

Non-Council Members: Drs B. Adams, A. Dora Black, B. Heine, J. Johnson, D. Kelly, D. Leigh, Miss $H$. Marshall, Miss F. Picken, Dr A. Walk.

Observer: Dr R. Jezzard (APIT)

\section{Journal Committee}

Council Members: Drs Valerie Cowie, P. d'Orban, D. Early, J. Evans, D. B. Gordon, O. Hill, I. S. Kreeger, E. G. Lucas, A. B. Sclare, A. Shapiro, B. Whitehead.

Non-Council Members: Drs H. R. Beech, G. E. Berrios, P. Bowden, Fay Fransella, J. Jancar, P. Tyrer.

Associate Editors: Dr A. Walk, Professor Sir Martin Roth.

Assistant Editors: Drs B. Barraclough, S. Crown, H. L. Freeman, T. A. Kerr, J. Leff, R. Levy, A. C. Smith, R. P. Snaith.

Observer: Dr C. Halstrom (APIT).

\section{Programmes and Meetings Committee}

Council Members: Professor T. Arie, Drs C. Davies, T. L. Dunn, A. M. P. Kellam, E. G. Lucas, W. R. McKibben, E. W. Shepherd, E. D. West.

Non-Council Members: Drs P. Bowden, J. Cobb, A. V. P. Mackay, B. L. Mallett, R. Murray, A. R. Nichol, C. M. B. Pare, Professor M. Shepherd, Dr A. C. P. Sims.

Observer: Dr J. Bird (APIT).

\section{Public Policy Committee}

Council Members: Drs H. Cameron, J. M. Cuthill, C. Davies, D. Early, H. Eaton, J. J. Fennelly, Professor J. Gunn, Drs J. R. Hamilton, J. Harrington, M. Markowe, T. Nelson, J. M. Roberts, P. Sainsbury, E. W. Shepherd, B. Ward, T. Gwynne Williams.

Non-Council Members: Drs W. A. L. Bowen, P. H. Connell, T. M. Cuthbert, J. E. McA. Glancy, T. A. Kerr, S. Levine, Professors A. K. M. Macrae, R. G. Priest.

Observers: Drs E. F. Carr (DHSS), R. A. W. Ratcliff (SHHD), J. Bird (APIT). 